\title{
CALCULATIONS OF HARD SPHERE PACKINGS IN LARGE CYLINDERS
}

\author{
B.E. CLANCY
}

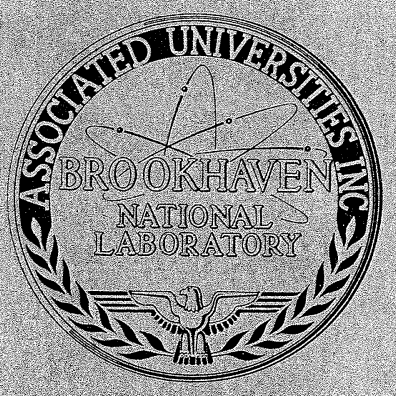

June 1966

BROOKHAVEN NATIONAL LABORATORY ASSOCIATED UNIVERSITIES, INC. under contract with the UNITED STATES ATOMIC ENERGY COMMISSION 


\title{
CALCULATIONS OF HARD SPHERE PACKINGS IN LARGE CYLINDERS
}

\author{
B.E. CLANCY*
}

June 1966

${ }^{*}$ On attachment from AAEC Research Establishment, Sutherland, N.S.W., Australia.

BROOKHAVEN NATIONAL LABORATORY UPTON, NEW YORK 11973 


\section{E G A L NOTICE}

This report was prepared as an account of Government sponsored work. Neither the United States, nor the Commission, nor any person acting on behalf of the Commission:

A. Makes any warranty or representation, expressed or implied, with respect to the accuracy, completeness, or usefulness of the information contained in this report, or that the use of any information, apparatus, method, or process disclosed in this report may not infringe privately owned rights; or

B. Assumes any liabilities with respect to the use of, or for damages resulting from the use of any information, apparatus, method, or process disclosed in this report.

As used in the above, "person acting on behalf of the Commission" includes any employee or contractor of the Commission, or employee of such contractor, to the extent that such employee or contractor of the Commission, or employee of such contractor prepares, disseminates, or provides access to, any information pursuant to his employment or contract with the Commission, or his employment with such contractor.

\section{PRINTED IN USA}

\section{PRICE $\$ 2.00$}

Available from the

Clearinghouse for Federal Scientific and Technical Information

National Bureau of Standards

U.S. Department of Commerce

Springfield, Virginia 
ABSTRACT

A procedure is described for generating numerical models of various arrangement of hard spheres packed in a cylindrical vessel. Calculations of a variety of configurations have been carried out on a digital computer for two packing models. The results are compared with measurements of the packing density in experimental configurations and with other measured properties of the packings. 
INTRODUCT ION

The nature of randomly packed configurations of hard spheres is of interest in a number of fields and has been studied for some time. Experimental measurements of overall packing density in a container (defined as the ratio of the volume of the spheres to the total container volume) have been made a number of times $(\underline{1}, \underline{2}, \underline{3})$ and reported in the literature and some measurements of other properties of the packings have been made $(\underline{4}, \underline{5}, \underline{6}, \underline{7})$. The experiment to measure the overall density is at least conceptually simple but determination of other properties requires the use of techniques which are tedious in the extreme. The ultimate experiment must be to measure the position of every sphere in a configuration since all the other properties of the packing can be deduced from this. This ultimate experiment has also been attempted.

With the advent of the high speed digital computer it has become possible to attempt to study the problem by numerical methods, and a number of interesting results (ㅇ, $\underline{9}, \underline{10})$ have been obtained. Most of the numerical methods known to the author have been dynamical in nature and have followed the motion of one or more hard spheres at a time. The procedure has essentially been to try to imitate the way in which an assembly of spheres would be packed in a real physical situation. This type of procedure has to contend with the difficulty that the positions (and velocities) of all the spheres in the configuration must be stored in the computer's memory which is limited and thus only small configurations can be followed in detail. 
The technique described in the present report was developed so as to be able to study large configurations and is feasible if only the final static configuration is of interest. Thus in characterizing a particular packing no attention is paid to the way in which the packing may have been produced - by pouring, shaking or not shaking, etc.

The technique follows from the observation that any configuration of hard spheres which can be produced by physical means, shaking, pouring, rolling or squeezing, can be copied by assembling an equivalent configuration of spheres one at a time. It is simple to prove this for a configuration of spheres in a cylinder. Conceptually one knows the position (or can measure it) of each sphere in the final packing and thus the spheres can be ordered by their height above the base of the cylinder. Then theoretically the configuration can be copied by taking an equal cylinder and sequentially placing the spheres into the equivalent positions from above in the order determined. For the purpose of this procedure gravity is "turned off", as it were, so that each sphere stays in place after insertion. This insertion in order of height can always be done since there can be no spheres already placed which get in the way. The one sphere at a time copying can certainly be done by following different sequences; to reproduce the configuration all that is needed is a strategy which will describe where each sphere is to be placed.

Such a strategy may simply embody a list of the exact numerical positions of the spheres, so as to copy a particular configuration, but may be a more general set of rules. Such a more general strategy might simply be as follows: "Find the lowest accessible sphere (i.e. one not buried beneath other 
spheres). Place a new sphere in contact with this lowest sphere and in a position as close to the wall as possible. Repeat for each sphere in turn". It seems reasonable to expect that such a procedure if followed consistently would yield a final configuration of a particular class and in fact any configuration could be made to correspond to the strategy which leads to its construction. A strategy thus essentially describes a packing model.

The present report gives an account of a procedure by which such a class of packing configurations can be studied and gives some results for two such classes.

\section{THE NOMOSS PROGRAM}

So as to be able to generate large configurations of spheres packed in a consistent fashion, the NOMOSS program was written. It is convenient at this stage to describe the logic behind the program as well as its limitations before going on to describe the results achieved.

The version of the program coded for this study generates packings in a right circular cylinder, and the only essential data required to begin the generation is the radius of this cylinder together with a description of the present status of an initial configuration of spheres (there need only be one). The meaning of the word status will be made more clear presently. The positions of these initial spheres may be specified by the user in any way desired, alternatively they may be specified as the last status achieved on a previous run and recorded on magnetic tape. This provides the facility to generate a configuration by a succession of computer runs, if convenient, or 
since this status tape will be uplated many times during a computer run, it also provides a means of recovering from a machine error.

The status of the configuration at any stage is completely described when the following information is known.

It is necessary to know the number NB of those spheres present in the cylinder, the positions of which determine directly the possible positions of the next sphere to be added to the configuration. If a sphere is completely buried by other spheres it is clear that the buried sphere is unimportant and will not directly affect the position of a new sphere. Buried spheres are thus not counted in computing the number NB. The coordinates $r_{i}(i=1,2 \ldots N B)$ of the centers of the important spheres must also be stored.

If an extra sphere were allowed to approach the configuration from above it would be in an equilibrium position (stability or otherwise not specified) if it were to be in contact with three of the important spheres $1,2 \ldots \mathrm{NB}$, and the central coordinates of these three spheres determine completely the position of the center of the additional sphere in this point of equilibrium or site. For each possible site "j" the central coordinates $r ! j$ are stored together with the identifying numbers of the three spheres which determine the position of the site. special treatment has to be given to those sites determined by two spheres and the curved wall of the cylinder or in the early stages of generating a configuration by sites determined partly by the position of the base of the cylinder. The total number NS of sites is also computed and stored. We note that many pairs of site centers will be closer together than a sphere diameter. on the other hand a sphere center must not be 
closer than this distance either to another sphere center or to a site center since the spheres cannot overlap. The last piece of information necessary to describe the status is a number NCOJw which for each important sphere gives the number of sites which the sphere helps to determine. It is in fact this count which enables the decision to be made whether or not a particular sphere is important. A buried sphere, and hence an unimportant one, is by definition one which a new sphere cannot reach; no possible sites for the new sphere can therefore be determined by the buried one. Important spheres are thus those which help to determine at least one site.

With the status of a configuration defined, the procedure followed by the Nomoss program is quite straightforward. All of the information determining the status is in storage and immediately accessible. To continue to generate the configuration it is necessary to add an additional sphere and the coordinates of this new sphere are selected by a particular subroutine ("SELECT") which also assigns an identification number to the sphere. The only a priori rules which must be satisfied by the SELECT routine are that the new sphere shall not overlap any of the NB important spheres already in the cylinder, shall not overlap the walls or base of the cylinder and shall be introduced into the cylinder from above. Different packing models were studied by coding the SELECT subroutine to perform the selection in a manner appropriate to the model. The presence of the additional sphere means that it and certain of the important spheres in the neighborhood will between them determine additional sites which are then computed and added to the list of sites. The new sphere, however, may overlap certain of the previously computed sites and these overlapped 
sites have to be removed from the list. When a site is removed the identification numbers of the spheres which determine that site are looked up and for these spheres the number NCOUNT of sites which they determine is decreased by one. As a site is added to the list, the reverse processes have to be carried out. After all these modifications have been made to the site list and the appropriate pieces of bookkeeping have been performed the NCOUNT array for the important balls is scanned to determine whether any of them have become buried and can therefore be reclassified as unimportant.

At this stage the major loop in the program has essentially been completed and any desired editing of the configuration can be performed. The version of the program coded wrote out at this point the coordinates of the new sphere, the total number of spheres in the cylinder and the packing density achieved with this latest sphere. Some additional edit calculations were made and the major loop was restarted by going back to the SELECT routine to choose yet another new sphere.

The program was coded for the IBM 7094 in a mixture of FORTRAN II and FAP. By using FAP coded routines it was possible to store in one machine word the identification numbers of the three spheres determining a site, and when necessary to unpack these three numbers. When this is done the main storage requirement for data is about $4(\mathrm{NB}+\mathrm{NS})$ where $\mathrm{NB}$ is the number of important spheres and NS the number of sites. This limits the size of cylinders which can be handled by the program to those with a diameter of not much more than 40 sphere diameters. The speed of the program is sufficient to load about 250 spheres per minute, a speed essentially independent of the size of the cylinder. The program spends most 
of its time in calculation of the coordinates of possible sites and relatively little time in the bookkeeping operations of keeping the list of sites compact (as sites are rejected), checking spheres to see whether they have been buried and so on. The time taken in computing site positions is reduced by doing preliminary searches to construct for the newly added sphere a short list of those important spheres which are within two ball diameters distance. This short list is then scanned to select posible spheres which could with the new sphere determine additional sites. Further improvements in the speed of the program would require very careful coding of the routines which actually compute the sites.

\section{PACKING MODELS}

Two packing models were examined in detail for the present study. For both of these models the procedure followed by the SELECT routine of the NOMOSS code was to choose for the position of the new sphere to be added one of the equilibrium sites whose positions had already been computed. For the first model studied, the random single packing model, a small circular. region of the surface of important spheres was chosen at random, the diameter of the subregion being three ball diameters and the lowest site in the region was chosen as the position of the new ball. The choice of a site as the locally lowest site ensures that the equilibrium for the site is stable and it was thought that the model might reproduce in some average sense those configurations produced when spheres are slowly and gently poured into a cylinder and not shaken down. For the second model studied the selection procedure was to choose the position of the new ball to be always 
at the very lowest equilibrium site. This model called optimum single packing was chosen since it would seem likely to produce the tightest packing of any model for which the new sphere is placed in an equilibrium site already computed.

The experimental results of scott (ㅁ) describe for cylinders of fixed diameter asymptotic densities for packings of large height. It is important to know whether such a limiting density exists independently of the way in which the first few spheres are placed in the cylinder. For both models a number of runs were made with different initial configurations and the limiting value was found to be independent of the initial state. The results of a pair of such runs are indicated in Fig. 1 where the curves shown are indicative of the nature of the results obtained for all cylinders. Each sequence of rectangles envelopes the oscillating curve of the packing density plotted against the inverse height of the cylinder, the unit of length being taken as one sphere diameter. Successive points on the curve are obtained as each ball is added to the configuration and the height of the cylinder enclosing it increases. The random nature of the packings is indicated by the variation observable in the density which is quite significant even for the large height used. This random variation is indicated by the height of the various rectangles. The longer period variations are caused by the occurrence of a number of regular layers of spheres near the plane base of the cylinders, but this effect does not continue for long. The curves in Fig. 1 represent the growth of configurations generated in a cylinder of diameter 30 units. ( 1 unit = sphere diameter) according to the optimum single packing model. The upper curve was produced from an initial configuration of two spheres touching one an- 
other and touching the base of the cylinder. This initial state produces a large region near the base of the cylinder where the packing configuration is the perfect configuration with a high density to start. The lower curve was produced from an initial configuration of approximately 150 spheres arranged in a loose array on the base of the cylinder and this produced looser packings initially. The curves show clearly. however, that this initial state is not affecting the limiting value of the density as the height tends to infinity and verifies the belief that a real asymptotic density exists. The curves show also that the problem of estimating this asymptotic value is not a simple one. Each of the configurations in Fig. 1 ultimately contained upwards of 20,000 spheres and the asymptotic density was estimated from a straight line fit to the curve of density: against inverse height for the last 1000 spheres added. Even when such a large number of spheres was used, the limiting density could barely be estimated to within $0.5 \%$.

\section{COMPARISON OE MODELS WITH EXPERIMENT}

Packing Models

Using the procedure outlined in the last section to estimate asymptotic densities, a number of configurations were generated for each of the single packing models. These were generated in cylinders with diameters ranging from 5.6 to 30 units and the results obtained are tabulated in Table 1 and shown in Fig. 2 where the densities are plotted for both models against the inverse diameter of the cylinders. Fitting a straight line to the plotted points enables an estimate to be made of the doubly asymptotic density in the limit as the diameter of the cylinders tends to infinity. Plotted in the same figure are 
the experimental curves of scott for the two packings he describes as loose random packing and close random packing. The existence of the computed curve for random single packing with density lower than that observed by scott suggests that a certain amount of shaking and settling may have occurred even in the loosest configurations observed by scott.

Most interesting, however, is the range of experimental configurations with densities greater than those produced by the optimum single packing model. Those packings produced by long shaking indicate that the packing model necessary to reproduce them will need to be more sophisticated than the single packing models examined in the present study. It seems that it is necessary to choose for some or all of the spheres added to the configuration a position which does not coincide with the equilibrium sites already computed as part of the status. For spheres placed in such a way to be stable it would be essential for spheres added at a later stage to supply the needed support. It would appear that the two packing models tested give theoretical configurations which adequately imitate the looser random packings so far as density is concerned at least. They may thus reasonably be expected to imitate the packings in other ways as well and a number of other properties of the theoretical configurations were computed. Where these other properties do not depend strongly on the packing density the theoretical figures may be expected to be adequate for the closer packings as well. 
Statistical Aspects of the Configurations

The problem of estimating the asymptotic packing density from the densities of cylinders of finite height has been mentioned earlier. As spheres are added sequentially to the cylinder by the Nomoss program, the density of the packing is computed and stored. When $M$ spheres are in the cylinder the density $\rho_{M}$ of the packing is then given by the expression:

$$
\rho_{M}:=\frac{2 M}{3 D^{2} h_{M}}
$$

where $\mathrm{D}$ is the diameter and $\mathrm{h}_{M}$ the height of the packing in the cylinder, both measured in units of a sphere diameter. The height $h_{M}$ will be one unit more than the difference between the heights of the highest and lowest spheres.

If an asymptotic density $\rho_{\infty}$ exists and has been reached at this point the addition of a further N-M balls will increase the height of the packing by $\left(h_{N}-h_{M}\right)$ where

$$
\rho_{\infty}=\frac{2}{3 D^{2}} \cdot \frac{N-M}{h_{N}^{-h}}
$$

The density at this point will be

$$
\rho_{N}=\frac{2 N}{3 D^{2} h_{N}}
$$

which combined with equations (1) and (2) implies that

$$
\rho_{\mathrm{N}}=\rho_{\infty}+\frac{\alpha}{\mathrm{h}_{\mathrm{N}}}
$$

where

$$
\alpha=h_{M}\left(\rho_{M}-\rho_{\infty}\right)
$$


and is thus independent of $\mathrm{N}$.

It is therefore to be expected that when $\rho_{N}$ is plotted against $1 / h_{N}$ the resulting curve should be essentially linear for large values of $N$. Because of the variability present in the configurations, however, such a curve has superimposed on it the random fluctuations which are enveloped by the rectangles drawn in Fig. 1. This fluctuating curve is found to be well described for large $\mathrm{N}$ by equation (3) provided a is interpreted as a random variate with mean equal to the value quoted in equation 4. For the cylindrical configurations studied, $\alpha$ appears to be distributed normally with variance approximately $0.03 / \mathrm{D}^{2}$. The parameters $\rho_{\infty}$ can thus be estimated by a least squares fit of equation (3) to the observed points on the curve and this calculation was performed by the program at regular stages until several successive estimates were in agreement to within the accuracy desired.

Radial Dependence of Local Density

The local density for any region of the cylinder is the proportion of the volume of the region occupied by spheres or parts of spheres. A quantity of interest which has been measured experimentally () for some packings is the local density $\rho(r)$ of cylindrical shells of thickness $\delta r$ co-axial with the cylinder in which the spheres are packed. From samples of 2000 spheres sufficient data can be accumulated to give reliable statistics for this local density as a function of the radius of the shells for about two ball diameters distance from the walls of the cylinder and for a cylinder of diameter 20.1 units this curve is shown in Fig. 3 . For shells nearer to the axis of the cylinder too few spheres are present for the curve to display anything more than random 
fluctuations. Near the wall, however, the oscillatory behavior associated with the ordering of the spheres is clearly seen with the first maximum at a distance of 0.7 units and the first minimum at 1.1 units. The second maximum and minimum are clearly visible but their positions cannot be clearly determined. The second maximum, however, certainly falls between 1.4 and 1.6 units distance from the wall. The curve in Fig. 3 is taken from a configuration produced using the optimum single packing model but the same shape is evidenced with the random single packing model. It is apparent that more reliable curves can be obtained but larger samples of spheres must be studied in order to do so.

The integrated radial density $\bar{\rho}(r)$ defined by

$$
\bar{\rho}(r)=\frac{2}{a^{2}-r^{2}} \int_{r}^{a} r^{\prime} \rho\left(r^{\prime}\right) d r^{\prime}
$$

in cylinders of radius 'a' was also measured by Benenati et al (7). The experimental curve for cylinders of diameter 20.3 units is compared in Fig. 4 with the curve for the optimum single packed model. The difference shows clearly that the experimental packing was far more strongly ordered near the cylinder walls than is the case for the single packing model studied.

Distribution of First Neighbors

The mutual coordination of packings of spheres has been studied by Bernal and Mason (ㄴ) who measured for a number of spheres in a configuration the number of points of contact with neighboring spheres by a very tedious process. This distribution of neighbors is simple to determine for the configurations generated by the NOMOSS program. This distribution is given in Table 2 for two samples of optimum 
single packing and one sample of random single packing. The difference in means between the two samples is not statistically significant at any level and because of the wide difference in densities produced by the two models it may be reasonable to assume that the form of the distribution will be maintained for more closely packed configurations. The means found for these samples are not inconsistent with the distributions reported by Bernal and Mason for close contact and also given in Table 2 . 


\section{ACKNOWLED GMENTS}

The author is grateful to Jack Chernick for many fruitful suggestions during the course of the work and to Bill

Bornstein for his invariably patient assistance in debugging the computer program. 
REFERENCES

1. Westman, A. E. R., and Hugill, H. R., J. Amer. Ceramic Soc., 13, 767 (1930).

2. Rice, O. K., J. Chem. Phys., 12, 1 (1944).

3. Scott, G. D., Nature 188, 908 (1960).

4. Bernal, J. D., and Mason, J., Nature 188, 910 (1960).

5. Scott, G. D., Nature 194, 956 (1962).

6. Scott, G. D., and Mader, D. L., Nature 201, 382 (1964).

7. Benenati, F. and Brosilow, C. B., A. I. Ch. E. Journal, 8. 359 (1962).

8. Levine, M. M., and Chernick, J., Nature 208. 68 (1965).

9. Alder, B. J.. and wainwright, T. E., J. Chem. Phys. $\underline{3}$, 1439 (1960).

10. Levine, M. M., BNL-10226 (1966) . 
Table 1

\begin{tabular}{|c|c|c|c|}
\hline $\begin{array}{l}\text { Packing } \\
\text { Model }\end{array}$ & $\begin{array}{l}\text { Diameter of } \\
\text { Cylinder* }\end{array}$ & $\begin{array}{l}\text { Number of Spheres in } \\
\text { Final Configuration }\end{array}$ & $\begin{array}{l}\text { Asymptotic } \\
\text { Density }\end{array}$ \\
\hline Random & 5.6 & 19.974 & .528 \\
\hline Single & 10.0 & 19,300 & .558 \\
\hline Packing & 30.0 & 18,327 & .581 \\
\hline Opt imum & 5.6 & 11.897 & .544 \\
\hline Single & 10.0 & 19,923 & .571 \\
\hline Packing & 20.1 & 17,791 & .588 \\
\hline & 30.0 & 29,034 & .593 \\
\hline
\end{tabular}

${ }^{*}$ Sphere diameter $=1.0$ unit. 


\section{Table 2}

Distribution of number of contacts with neighboring spheres

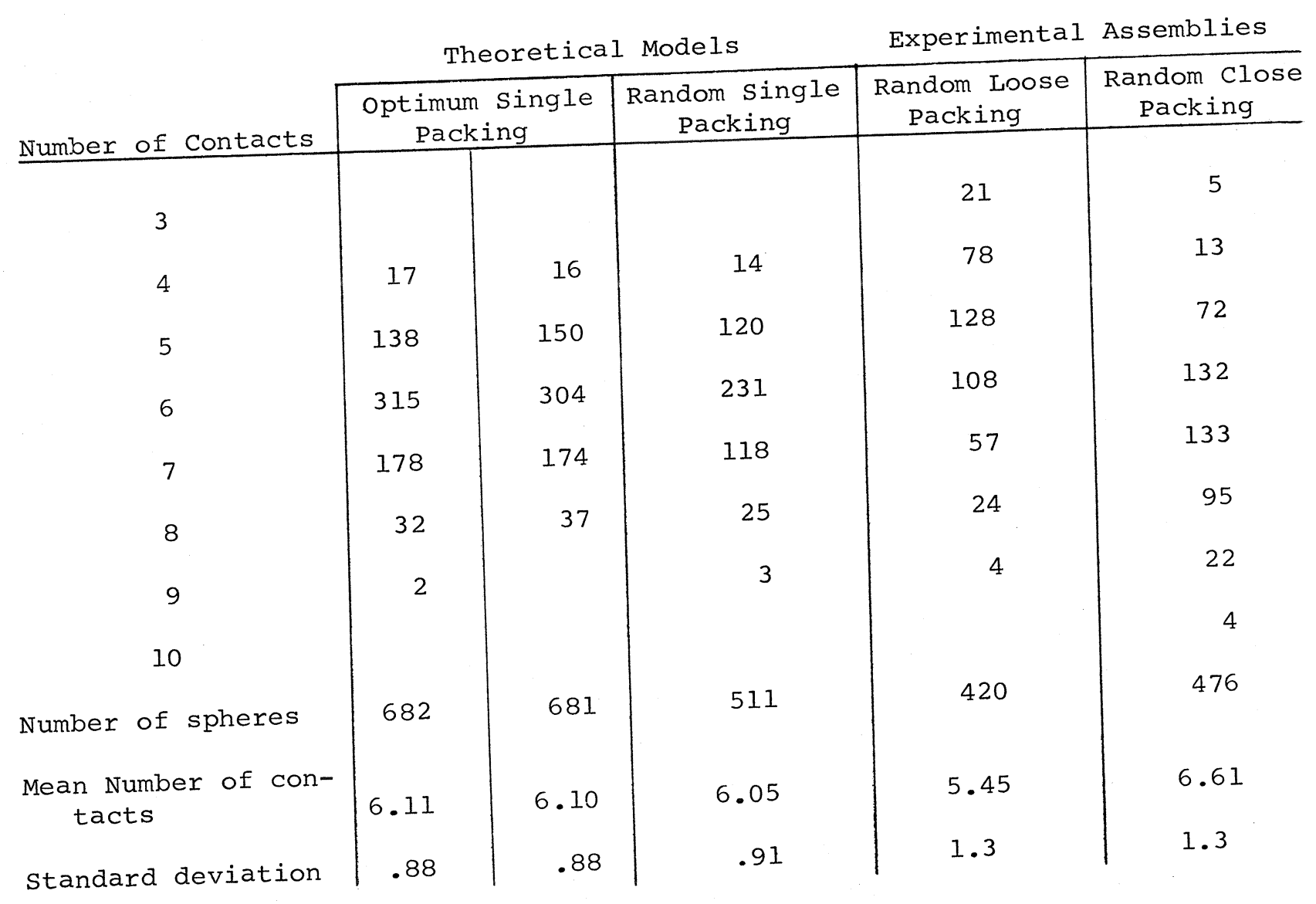




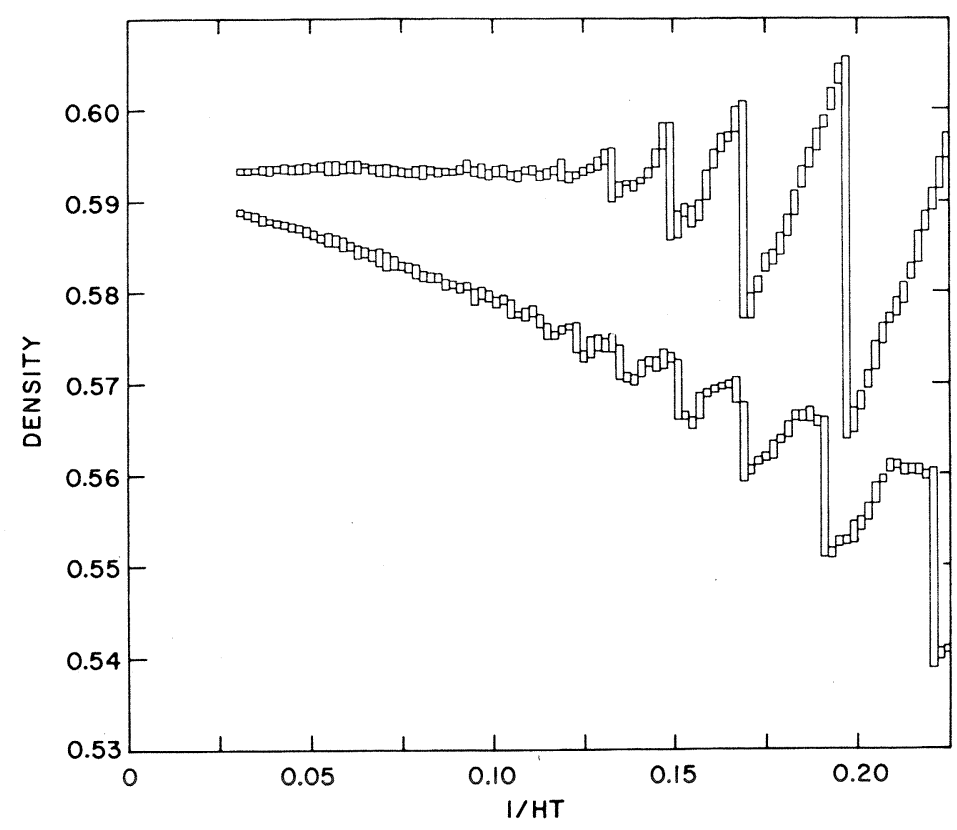

FIGURE 1

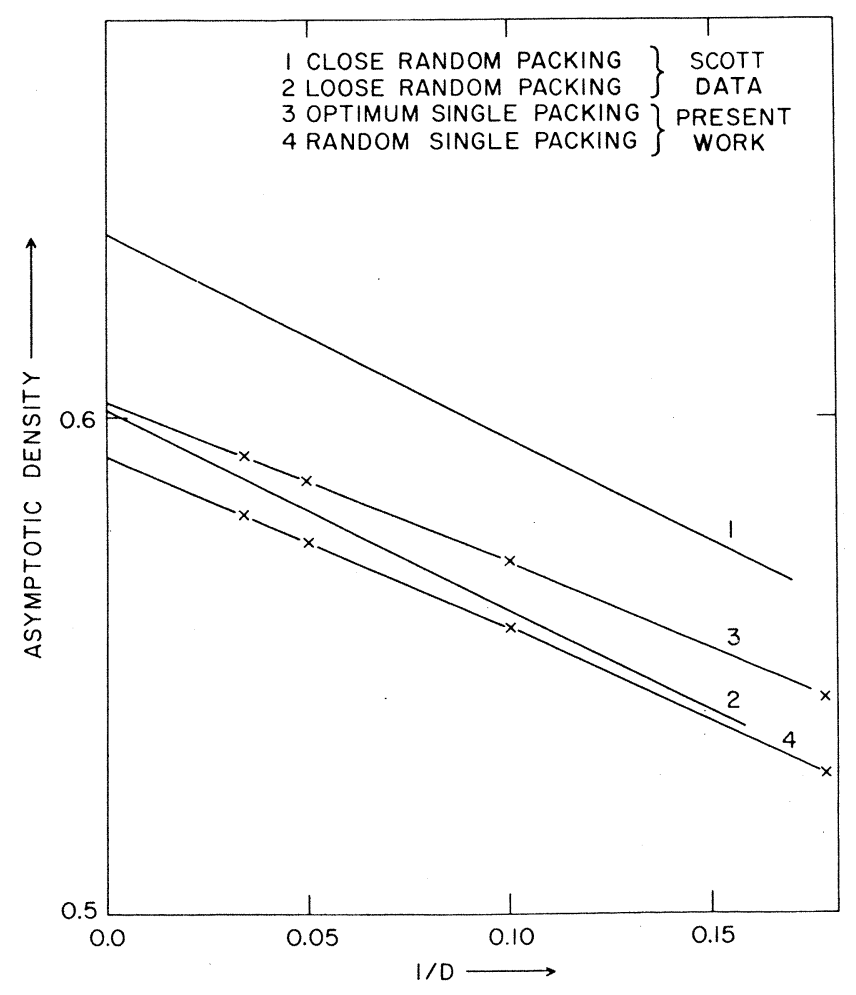

FIGURE 2 

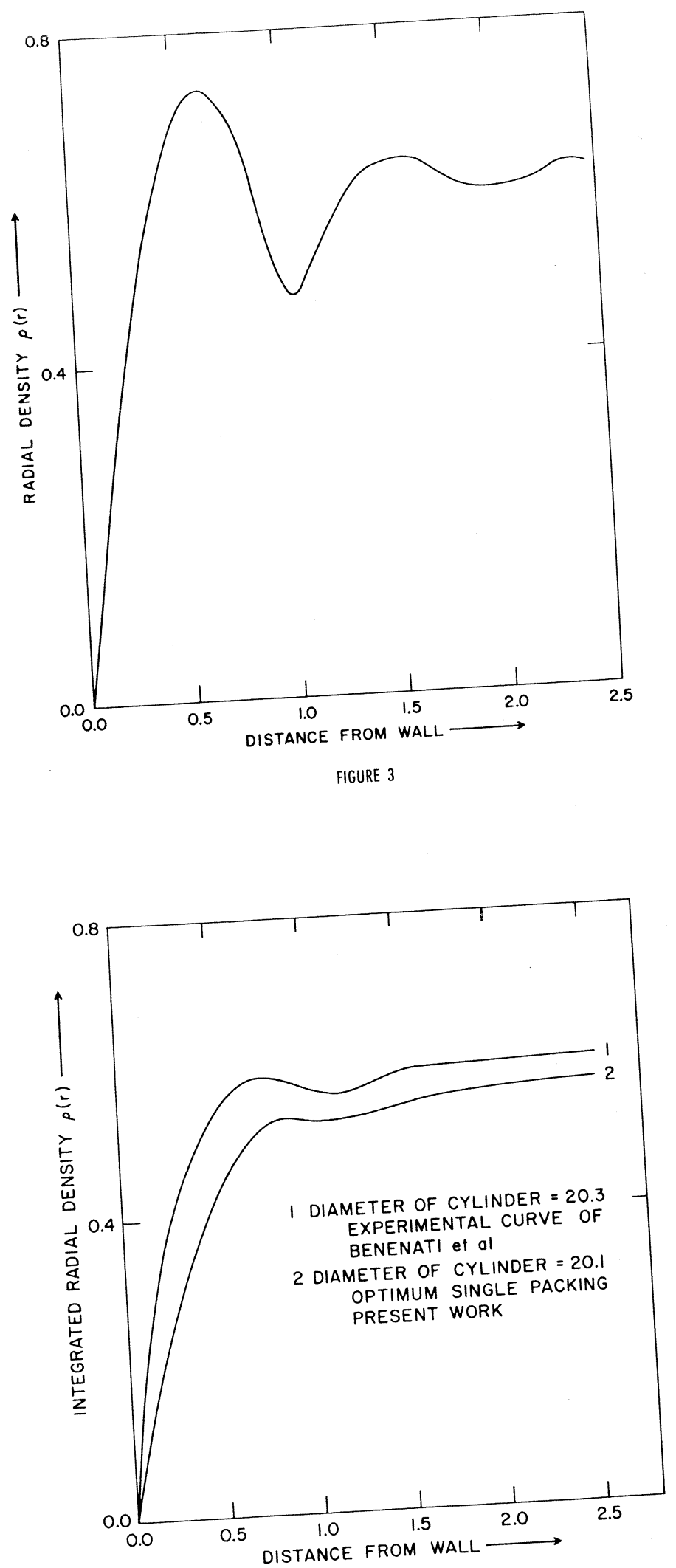

FIGURE 4 